January 6, 1996

\title{
LATTICING QUANTUM GRAVITY ${ }^{1}$
}

\author{
R. Loll \\ Max-Planck-Institut für Gravitationsphysik \\ Schlaatzweg 1 \\ D-14473 Potsdam, Germany \\ and \\ Erwin Schrödinger Institut \\ Boltzmanngasse 9 \\ A-1090 Wien, Austria
}

\begin{abstract}
I discuss some aspects of a lattice approach to canonical quantum gravity in a connection formulation, discuss how it differs from the continuum construction, and compare the spectra of geometric operators - encoding information about components of the spatial metric - for some simple lattice quantum states.
\end{abstract}

To appear in the Proceedings of the 2nd Conference on Constrained Dynamics and Quantum Gravity, Santa Margherita, Italy, 17-21 September 1996. 


\section{Discretizing gravity}

My contribution describes some aspects of an attempt to define a quantum theory of gravity in $3+1$ dimensions, starting from a lattice discretization of spatial 3-manifolds. This approach is complementary to other ones currently under study, most importantly, the Regge calculus program and its variant, using so-called dynamical triangulations. It differs from them in at least two aspects. Firstly, its basic configuration variables are not discretized versions of the space- or space-time metric tensor, but of $s u(2)$-valued connection one-forms. Secondly, in order to exploit the structural resemblance with lattice gauge field theory, one best uses Hamiltonian, and not path integral methods.

\subsection{Setting}

The classical starting point is a reformulation of Einstein gravity in terms of a phase space variable pair $\left(A_{a}^{i}(x), E_{i}^{a}(x)\right)$ defined on a continuum 3-manifold $\Sigma$, where $a$ is a spatial and $i=1,2,3$ an adjoint $s u(2)$-index. This is a real version of the well-known $s u(2, C)$-valued Ashtekar variables (but still describing Lorentzian, and not Euclidean gravity!). Using the real variables, one avoids the difficulty of having to impose quantum reality conditions, but the Hamiltonian constraint acquires a potential term which is not present in the complex formulation. This is functionally rather involved, but can probably still be handled [1]. The (doubly densitized, inverse) metric tensor is a function of the momentum variables, $g^{a b}=E_{i}^{a} E^{b i}$.

In terms of the $(A, E)$-variables, Einstein gravity assumes the form of a Dirac constrained system, subject to a set of seven first-class constraints per space point, namely, three spatial diffeomorphism, one Hamiltonian and three Gauss law constraints.

In the lattice approach [2], one approximates $\Sigma$ by a lattice $\Lambda$, consisting of one-dimensional edges or links $l_{i}$ meeting at vertices $n_{j}$. For simplicity, $\Lambda$ is chosen cubic, and all vertices are of valence six. The lattice analogues of the Hamiltonian variables $(A, E)$ are a set of link-based variables $(V, p)$ which however are not canonical. This comes about because the link analogue of the local algebra-valued connection $A(x)$ is the group-valued exponentiated integral of $A$, the link holonomy $V_{a}^{B}(l)$. Hence the configuration space associated with a single link is a copy of the compact group manifold $S U(2)$.

The wave functions of the quantum theory are the square-integrable functions on the

product over all links of the group $S U(2)$. The operators $\hat{V}_{a}^{B}(l)$ are represented by multiplication and the non-local link momenta $\hat{p}(l)$ can be identified with the left- and right-invariant 
vector fields on the l'th copy of the group.

The kinematical structure described above is identical with the one used in Hamiltonian lattice gauge theory. This setting is also well-suited for gravity, since the part of the constraints corresponding to internal gauge rotations is identical with those of Yang-Mills theory. One has two choices of dealing with the gauge constraints: one can either keep discretized versions of the quantum Gauss law constraints and eventually use them to project out physical, gauge-invariant wave functions, or go directly to a basis of gauge-invariant quantum states. We will follow the latter path, but this choice is not substantial.

The elementary functions spanning the gauge-invariant Hilbert subspace $\mathcal{H}^{\text {inv }}$ are the traces of holonomies of closed lattice paths, obtained by multiplying together the corresponding link holonomies. An independent basis can be given in terms of so-called spin-network states, where one assigns unitary irreducible representations of $S U(2)$ (i.e. half-integer spins) to links and gauge-invariant contractors to lattice vertices. However, the reader should be warned that in explicit calculations one still has to worry about the presence of zero-norm states, that exist in the form of Mandelstam constraints. Equivalently, the choice of an independent set of states involves - for fixed spin assignments - a selection of independent contractors from the entire set at each vertex $n$. For each $n$, the spaces involved are finitedimensional, but their dimension grows fast for increasing spins.

\subsection{Lattice vs. continuum theory}

The lattice construction is in many aspects similar to the loop quantization program in the continuum, that also uses $S U(2)$-valued holonomies $U_{\gamma}[A]:=\mathrm{P} \exp \oint_{\gamma} A$ or their traces $\operatorname{Tr} U_{\gamma}[A]$ as the basic configuration variables. However, in order to avoid confusion, let us point out the main differences between the two formalisms.

Graph or lattice configurations also appear in the continuum theory as part of the specification of a quantum state. However, to obtain the entire Hilbert space of the kinematical quantum theory (i.e. before imposition of the Hamiltonian and diffeomorphism constraints), one has to consider states associated with all possible graphs. As a consequence, in order to specify a quantum state completely, one needs a) a graph $\gamma$, b) consistent non-vanishing spin assignments to all of its edges, and c) matching gauge-invariant contractors at all non-trivial vertices of $\gamma$. Of course, linear combinations of such states are also possible. The Hilbert space is, loosely speaking, the space $L^{2}(\mathcal{A} / \mathcal{G})$ of all square-integrable functions on the space of gauge connections modulo gauge, which is an infinite-dimensional space. 
By contrast, the configuration space for lattice gravity (for a finite lattice) is finitedimensional. Before considering gauge transformations, there are three degrees of freedom associated with each lattice link (parametrizing an element of $S U(2)$ ) times the number of links of the lattice. Furthermore, the lattice is fixed, i.e. all states and operators are defined on the same lattice. (Eventually, one wants to make the lattice bigger, in order to obtain a better approximation to the continuum theory. Still, the lattice operators never mix states associated with different lattices.) In order to specify a quantum state on the lattice, one needs a) consistent spin assignments to all of its edges (vanishing spin is allowed, but does not imply that the underlying link "vanishes"), and b) matching gauge-invariant contractors at all lattice vertices.

A related important difference is that the continuum states depend on graphs imbedded in $\Sigma$, whereas lattice states are based on a subset of lattice links, with the lattice $\Lambda$ itself not thought of as imbedded in an underlying manifold, but as a discrete approximation to $\Sigma$. As a result, in the continuum theory we can still define an action of the group of diffeomorphisms $\operatorname{Diff}(\Sigma)$ on states in a straightforward way. The lattice theory does not possess enough degrees of freedom to support such an action, and the most one can hope for is to define some kind of "discrete version of $\operatorname{Diff}(\Sigma)$ ", that goes over to the usual continuum action in the limit as the lattice spacing $a$ is taken to zero. This is a non-trivial issue also in other discrete approaches to quantum gravity.

Note that the appearance of one-dimensional "loopy" excitations in the lattice theory is a consequence of the type of discretization we have chosen, and should not necessarily be considered fundamental, in the sense that as the continuum limit is approached, one may expect only genuine three-dimensional properties of states and operators to be physically important. The central assumption of the lattice construction is of course the existence of such a continuum limit.

On the other hand, the fundamental assumption that leads to the continuum loop representation is that the Wilson loops $\operatorname{Tr} U_{\gamma}[A]$ become well-defined operators in the quantum theory. Classically, the information contained in the $\operatorname{Tr} U_{\gamma}$ allows one to reconstruct the space of smooth connections modulo gauge. Quantum-mechanically, the operators $\hat{\operatorname{Tr}} U_{\gamma}$ can be thought of as distributional excitations of the connection $A$ along some loop or graph $\gamma$, and are therefore rather singular objects from a three-dimensional point of view. Nevertheless, well-behaved unitary representations of the classical algebra of the Wilson loops exist, and it is exactly those that have been used in the loop quantization approach. They do have some peculiar properties, for example, operator actions tend to be sensitive to certain topological characteristics of quantum loop states, such as their number of edges or vertices, 
and the way flux lines are arranged. However, this is certainly not the only way one may set up a quantum theory. It has been argued that it is physically more realistic to quantize configuration variables associated with three-dimensionally smeared objects, like for instance tubes instead of loops. Quantization of such an algebra is not likely to share all of the features that have made the loop representations so attractive.

\section{Geometric operators}

In spite of the differences outlined in the previous section between the lattice and the continuum quantum theories, there obviously is a great structural resemblance between the two. This is in particular true for the action of certain geometric operators one may construct in both settings, measuring volumes, areas, and lengths of spatial regions. It is not my aim here to discuss the construction and properties of these quantities in great detail, but rather to focus attention on a point that has not yet been addressed much in the literature.

The classical continuum expressions for volume, area and length are given purely as functions of the inverse dreibein variables $E$,

$$
\begin{gathered}
\mathcal{V}(\mathcal{R})=\int_{\mathcal{R}} d^{3} x \sqrt{\frac{1}{3 !} \epsilon_{a b c} \epsilon^{i j k} E_{i}^{a} E_{j}^{b} E_{k}^{c}}, \\
\mathcal{A}(\mathcal{S})=\int_{\mathcal{S}} d^{2} x \sqrt{E^{3 i} E_{i}^{3}} \\
\mathcal{L}(\mathcal{C})=\int_{\mathcal{C}} d x \sqrt{\frac{E_{j}^{2} E^{2 j} E_{k}^{3} E^{3 k}-\left(E^{2 j} E_{j}^{3}\right)^{2}}{\operatorname{det} E}},
\end{gathered}
$$

where $\mathcal{R}$ is a three-dimensional spatial region, $\mathcal{S}$ a surface with unit normal in 3-direction, and $\mathcal{C}$ a curve dual to the 2-3-plane. As usual, their discretizations are not unique. We choose them as follows [3]:

$$
\begin{gathered}
\mathcal{V}\left(\mathcal{R}^{\text {latt }}\right)=\sum_{n \in \mathcal{R}^{\text {latt }}} \sqrt{\frac{1}{3 !} D(n)} \\
\mathcal{A}\left(\mathcal{S}^{\text {latt }}\right)=\sum_{n \in \mathcal{S}^{\text {latt }}} \sqrt{\frac{1}{2}\left(p_{i}^{+}(n, \hat{3}) p^{+i}(n, \hat{3})+p_{i}^{-}(n, \hat{3}) p^{-i}(n, \hat{3})\right)}
\end{gathered}
$$




$$
\mathcal{L}\left(\mathcal{C}^{\text {latt }}\right)=\sum_{n \in \mathcal{C}^{\text {latt }}} \sqrt{\frac{3 !}{D(n)}\left(p_{i}(n, \hat{2}) p^{i}(n, \hat{2}) p_{j}(n, \hat{3}) p^{j}(n, \hat{3})-\left(p_{i}(n, \hat{2}) p^{i}(n, \hat{3})\right)^{2}\right)}
$$

with $D(n)=\epsilon_{a b c} \epsilon^{i j k} p_{i}(n, \hat{a}) p_{j}(n, \hat{b}) p_{k}(n, \hat{c})$, and the symmetrized link momenta $p_{i}(n, \hat{a})$.

A nice property of the geometric lattice functions is that the expressions under the square roots can be represented by self-adjoint operators in the quantum theory, and therefore the operator square roots be defined in terms of the spectral resolutions. The spectra and eigenfunctions can be computed explicitly, by virtue of the fact that the quantum operators are defined purely in terms of the link momenta $\hat{p}$, which have a particularly simple action on spin-network states. A further consequence is that the diagonalization of geometric operators can be performed independently at each lattice vertex (operators associated with different vertices commute), which vastly simplifies their discussion.

The complete spectrum of the area operator $\hat{\mathcal{A}}$ can be written down immediately, since $\hat{\mathcal{A}}$ is a function of Laplacians only. The spectrum of the volume operator $\hat{\mathcal{V}}$ is only partially known, although general formulas for its matrix elements can be given. The spectrum of the length operator $\hat{\mathcal{L}}$ has not been studied yet.

One reason for investigating the geometric operators is their simplicity, as compared to that of the Hamiltonian constraint. In addition, knowledge of the spectrum of the volume operator is vital for constructing phase space functions depending in some way on density factors of the form $\sqrt{\operatorname{det} g} \equiv \sqrt{\operatorname{det} E}$, for example, the length function or the Hamiltonian.

We will compare the spectra of these operators for some simple, explicit spin-network configurations. Since unit cells of the lattice can be regarded as the smallest building blocks of geometry, one would certainly like to check whether the order of magnitude of the eigenvalues is comparable. To simplify matters further, we will consider maximally symmetric local lattice configurations, where no lattice direction is preferred, and concentrate on the volume and area operators.

Recall that a local spin-network configuration around a vertex $n$ is determined by assigning half-integer spins $s_{i}$ or flux line numbers $j_{i}=2 s_{i}$ to each of the six incoming lattice edges, and a gauge-invariant contractor at $n$. We take all six $j_{i}$ equal, $j_{i}=j, j=1,2, \ldots$ Fig.1 shows the length scales extracted from the area and (the non-negative) volume eigenvalues, i.e. the square and third root respectively. For the volume eigenvalues, the degeneracy of the eigenspace is indicated. The area eigenspaces are maximally degenerate. 


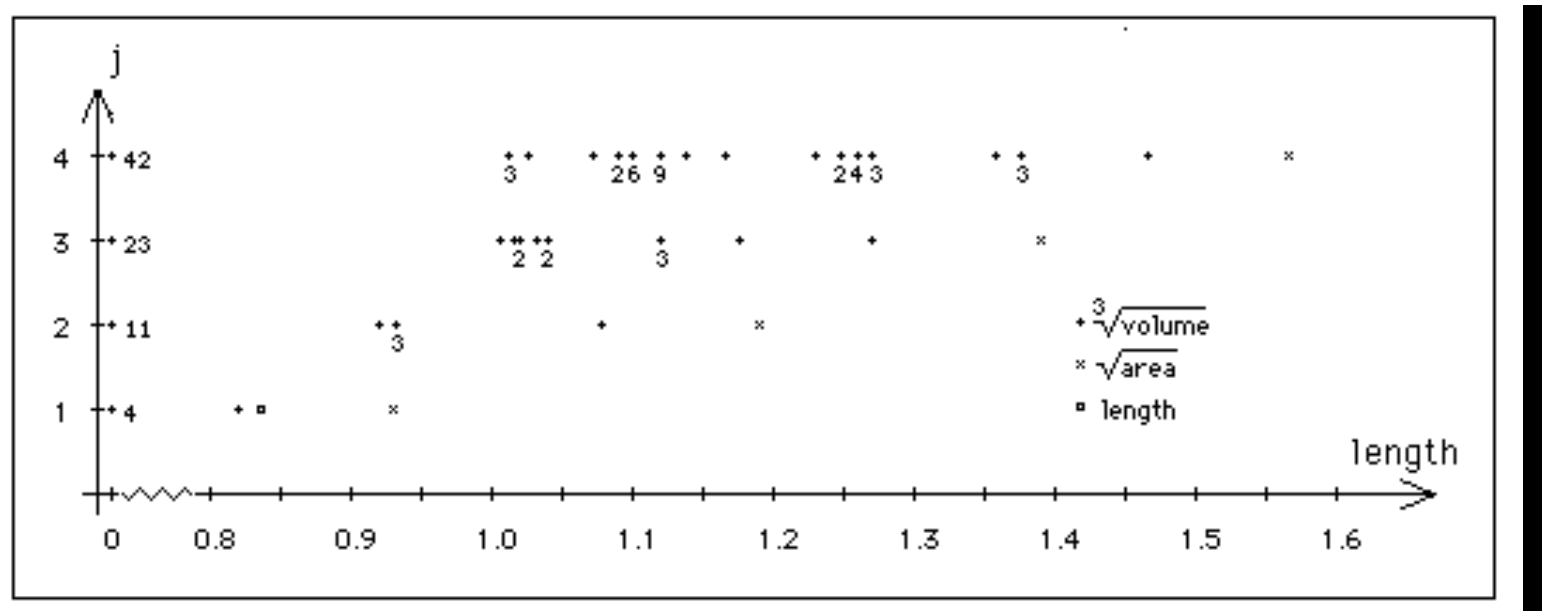

Fig.1 Eigenvalues of geometric lattice operators at a given vertex $n$.

Observe first that the length scales for given $j$ are roughly the same. This is also true for the single length eigenvalue calculated so far (it is that of the only positive-volume eigenstate for $j=1$ ). Beyond that, note that lengths obtained from the volume operator are systematically smaller than those obtained from the areas. This indicates that one may encounter problems when attempting to construct a macroscopic flat, Euclidean geometry from these microstates, even if one uses eigenstates maximizing the volume for given $j$. It is possible that this effect goes away for larger $j$. Alternatively, this "volume deficit" may be an indication that generic local geometries have a small non-vanishing scalar curvature (I thank S. Carlip for this suggestion).

Note also that the volume spectrum becomes more spread out with increasing $j$ and that there are many zero-volume states. An important issue in quantum gravity is whether or not these states can or must be included in the Hilbert space.

\section{References}

[1] R. Loll, Phys. Rev. D54 (1996) 5381; T. Thiemann, Phys. Lett. B380 (1996) 257.

[2] R. Loll, Nucl. Phys. B444 (1995) 619; Nucl. Phys. B460 (1996) 143.

[3] R. Loll, AEI preprint, Dec 1996. 